\title{
Coiled-coil intermediate filament stutter instability and molecular unfolding
}

Q1

\author{
Melis Arslan ${ }^{\mathrm{a}}$, Zhao Qin ${ }^{\mathrm{a}}$ and Markus J. Buehler ${ }^{\mathrm{a}, \mathrm{b}, \mathrm{c} *}$
}

${ }^{a}$ Laboratory for Atomistic and Molecular Mechanics, Department of Civil and Environmental Engineering, Massachusetts Institute of Technology, 77 Massachusetts Avenue Room 1-235A\&B, Cambridge, MA, USA; ${ }^{b}$ Massachusetts Institute of Technology, Center for Materials Science and Engineering, 77 Massachusetts Avenue, Cambridge, MA, USA; ${ }^{c}$ Massachusetts Institute of Technology, Center for Computational Engineering, 77 Massachusetts Avenue, Cambridge, MA, USA

(Received 12 November 2010; final version received 1 February 2011)

\begin{abstract}
Intermediate filaments (IFs) are the key components of cytoskeleton in eukaryotic cells and are critical for cell mechanics. The building block of IFs is a coiled-coil alpha-helical dimer, consisting of several domains that include linkers and other structural discontinuities. One of the discontinuities in the dimer's coiled-coil region is the so-called 'stutter' region. The stutter is a region where a variation of the amino acid sequence pattern from other parts of the alpha-helical domains of the protein is found. It was suggested in earlier works that due to this sequence variation, the perfect coiled-coil arrangement ceases to exist. Here, we show using explicit water molecular dynamics and well-tempered metadynamics that for the coil2 domain of vimentin IFs the stutter is more stable in a non-alpha-helical, unfolded state. This causes a local structural disturbance in the alpha helix, which has a global effect on the nanomechanics of the structure. Our analysis suggests that the stutter features an enhanced tendency to unfolding even under the absence of external forces, implying a much greater structural instability than previously assumed. As a result it features a smaller local bending stiffness than other segments and presents a seed for the initiation of molecular bending and unfolding at large deformation.
\end{abstract}

Keywords: intermediate filaments; alpha helix; coiled coil; nanomechanics; protein; materiomics

\section{Introduction}

Intermediate filaments (IFs) are a major building material of the cytoskeleton in eukaryotic cells, and are crucial in the determination of cell mechanical properties (Hutchison 2002; Herrmann and Aebi 2004; Herrmann et al. 2007). IFs are crucial in defining key functions of cells such as cell migration, cell division and mechanotransduction, and it is now understood that they play a critical role similar to other cytoskeleton elements such as microtubules and microfilaments. Notably and pointing to their rather important role as mechanical elements, IFs have also been referred to as the 'safety belts of cells', and have been demonstrated to play a role in preventing exceedingly large cell stretch (Wang et al. 1993; Wang and Stamenovic 2002; Fudge et al. 2008). A range of studies published over the past years focused on the mechanical signature of IFs, which have suggested that they can sustain extremely large deformation of up to several 100\% (Lewis et al. 2003; Herrmann et al. 2007; Kreplak et al. 2008). It was also found that due to severe stiffening, the tangent modulus of IFs increases manifold during deformation, which clearly could be crucial for providing mechanical resistance against large stretching. In addition to the cell's cytoskeleton, IFs are also found in the cell nucleus in the form of lamin IF, where they form a dense mesh-like network providing mechanical integrity and biochemical functions at the cytoskeleton-chromatin interface (Wilson et al. 2001; Dahl et al. 2004; Ackbarow et al. 2009). Numerous diseases associated with IFs were identified which point to the clear importance of material factors in the initiation and progression of these disorders (Brenner et al. 2001; Omary et al. 2004; Buehler and Yung 2009).

The basic building block of all IF proteins is a particularly stable molecular configuration of $\mathrm{H}$-bonded alpha-helix-based protein structures called coiled coils (Alberts 2002; Rose and Meier 2004). This arrangement of two alpha helices into coiled-coil domains provides increased strength to the structures. In alpha-helix-basedcoiled coils within a single heptad repeat (abcdefg periodicity), some positions are occupied by hydrophilic (polar) and some are occupied by hydrophobic (non-polar) residues (Alanine (ALA), Isoleucine (ILE), Leucine Q2 (LEU) ... ) (Strelkov et al. 2003; MacCallum et al. 2007; Q3 Buehler and Ackbarow 2008). The hydrophobic residues constitute the reason why these proteins assemble into coiled-coil structures as they ensure minimal contact of hydrophobic domains with water.

Vimentin IFs are the most widely distributed types among all IFs (Alberts 2002) and will be the focal point of the study reported here. The structure is made up of 310 amino acid residues consisting of four segments: 1A, 1B, 2A and 2B divided by three linkers: L1, L12, L2 as shown in

\section{*Corresponding author. Email: mbuehler@mit.edu}

ISSN 1025-5842 print/ISSN 1476-8259 online

(C) 2011 Taylor \& Francis

DOI: $10.1080 / 10255842.2011 .560147$

http://www.informaworld.com 
Figure 1(A). Segment 2A (residues 264-282) is the shortest one among all segments. It assumes the configuration of a nearly parallel alpha-helical bundle, in agreement with earlier suggestions (Parry et al. 2007; Qin et al. 2009; Nicolet et al. 2010). Segment 2B with 115 amino acids (residues 291-405) features alpha-helical coiled-coil geometry for the major segment. One of the discontinuities found in heptad repeats of an alpha-helical coiled-coil protein is the 'stutter' region. As a result it has been suggested that the molecular structure may be altered in this region and deviate from the perfectly coiled regions in the rest of the protein filament. The $2 \mathrm{~B}$ segment of vimentin contains the stutter region as visualised in Figure 1(A) (Brown et al. 1996; Strelkov et al. 2001; Ackbarow and Buehler 2009). It can be seen that the stutter is located at the end of the eighth heptad, in the vicinity of residue 351 (Strellkov 2002). An analysis of the amino acid sequence revealed that the nature of the vicinity of the stutter region is hydrophilic. Earlier work suggested that this results in a parallel arrangement for the two coils in the alpha helix rather than a coiled-coil configuration.

\section{Materials and methods}

\section{Molecular model construction}

Recent advances in identifying the structure of vimentin dimers with atomistic details (Strelkov et al. 2002; Nicolet et al. 2010), combined with protein simulation tools, now enable us to build molecular mechanics models of proteins at relatively large scales, involving tens of nanometres and with full atomistic resolution. Importantly, the development of this large model based directly on X-ray diffraction data is an advance over earlier smaller models in which the stutter was placed closer to the boundary of the filament (Ackbarow and Buehler 2009). The vimentin coil2 segment used here is obtained by combining two coiled-coil segments obtained directly from Protein Data Bank (PDB). The N-terminal half of coil2 is obtained from vimentin segment that includes residues 264-335 (obtained from the PDB with identification (ID) 3KLT Nicolet et al. 2010). The C-terminal half of coil2 is obtained from the vimentin segment that includes residues 328-406 (given by PDB with ID 1GK4 Strelkov et al. 2002). The two segments are aligned to form an integrated coiled coil because both fragments include an overlapped region of residues 328-335 as shown in Figure 1(B). We use visual molecular dynamics (Humphrey et al. 1996) to find the best fit between the backbone atoms of the overlapped region of the two segments corresponding to the overlapped part. The best fit provides a minimum root mean square deviation of $0.68 \AA$ between the C-alpha atoms within the overlapped region of the two segments as shown in Figure 1(C). We find that this result is better than directly superimposing the two segments (of $1.3 \AA$ ), and both of them indicate that the two segments match well with each other.

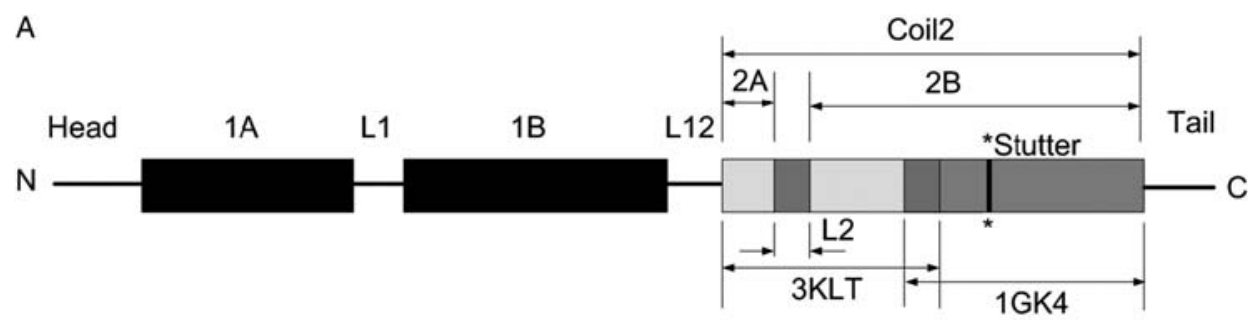

B
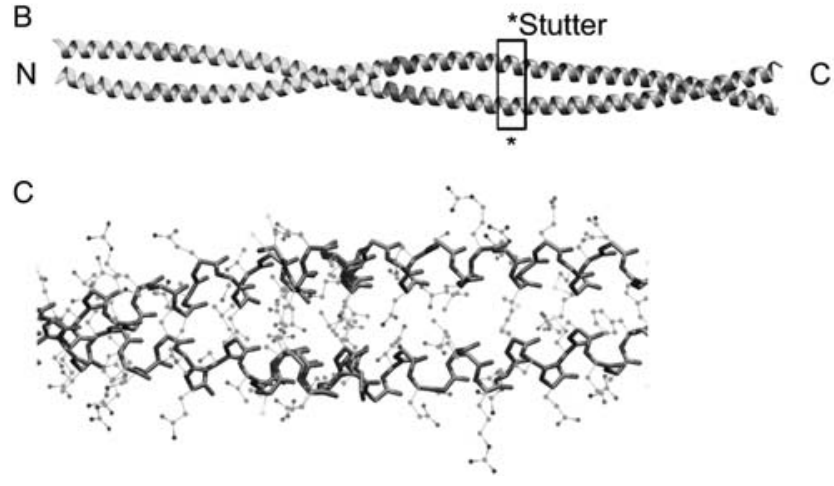

Figure 1. Schematic view of human vimentin dimer and the used segments in this study, with the location of the stutter region pointed out by the $*$ symbol. Panel (A): schematic view of the human vimentin IF dimer. Panel (B): cartoon view of the entire coil 2 domain in the vimentin dimer. Note that in Panels (A) and (B) the red regions correspond to the overlapped region (residues 328-335) as we use to integrate the two segments from PDB. Panel (C): detailed view at the overlapped region of the two segments after alignment. 


\section{Molecular simulation approach}

We use a standard explicit water molecular dynamics approach to first carefully equilibrate and then mechanically deform the coil 2 of vimentin. The structure is $21 \mathrm{~nm}$ long in its initial configuration. All molecular simulations are carried out using the CHARMM force field (MacKerell et al. 1998) as implemented in NAMD (Nelson et al. 1996). We use a 2 fs time step at a constant temperature of $T=300 \mathrm{~K}$ within a NPT ensemble. A pressure of $P=1$ atm is used. We start the simulation protocol by equilibrating the molecular structure for $20 \mathrm{~ns}$, where Figure 2 shows the resulting structure, including the constituent domains and the hydrophobic regions. We find that the $20 \mathrm{~ns}$ equilibration, limited due to computational constraints, gives a well-equilibrated structure with good geometry convergence. The molecular configuration obtained after equilibration is subsequently deformed using steered molecular dynamics (SMD) (Lu et al. 1998). In the simulation to deform the structure using SMD, the $\mathrm{C}$-alpha atoms at the head of the structure for both constituting chains are fixed whereas the $\mathrm{C}$-alpha atoms at the tail of the structure are pulled up to a strain of $30 \%$. The SMD spring constant used is $k=10 \mathrm{kcal} \mathrm{mol}^{-1} \AA$ in agreement with earlier studies. Varied pulling rates of 0.5 , $1.0,5$ and $10 \mathrm{~m} / \mathrm{s}$ are considered to present the validity of the approach and as a comparison with the force levels observed in earlier studies.

A
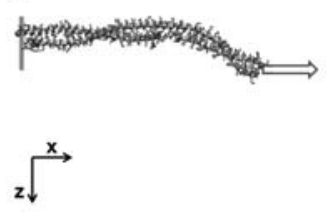

B

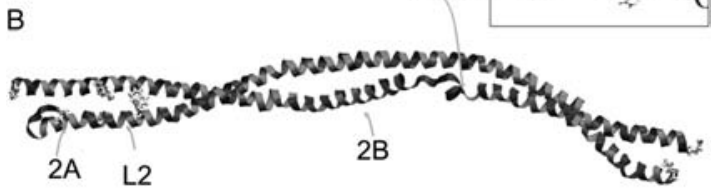

Hydrophilic Region
Hydrophobic Region

Figure 2. Panel (A): in the simulation that focuses on deforming the structure under uniaxial strain, the C-alpha atoms at the head of the molecule for both the constituent chains are fixed, whereas the $\mathrm{C}$-alpha atoms at the tail of the structure are pulled. Panel (B): the equilibrated structure with the constituent segments marked (2A, L2 and 2B) with an inset showing the detailed view of the stutter region. The structure was equilibrated for $20 \mathrm{~ns}$ in explicit water solvent and the $2 \mathrm{~A}$ region is found to be in a relatively rigid and parallel configuration, whereas the $2 \mathrm{~B}$ region is not aligned with any orthogonal axis and has gone through local deformations, especially at the vicinity of the stutter region which locally unfolds even without any applied force.

\section{Free-energy calculations}

In addition to SMD simulations, free-energy calculations are carried out using well-tempered metadynamics (Barducci et al. 2008) implemented in the PLUMED (Bonomi et al. 2009) plugin for NAMD, also at $T=300 \mathrm{~K}$. Tests are performed for two different sections in the $2 \mathrm{~B}$ domain in order to identify their free energy in the folded and unfolded state. The regions studied include the stutter and a set of residues neighbouring the stutter region that is known to form a stable alpha helix. The collective variable in metadynamics is chosen to be the distance between the C-alpha atoms of residues 349-353 for the stutter, and 363-367 for the other section. The tests are run for an initial configuration of perfectly folded alpha helix and also for the configuration obtained after equilibration for $20 \mathrm{~ns}$. The PLUMED runs here are $5 \mathrm{~ns}$ long and the convergence of the tests is confirmed.

\section{Results and discussion}

We begin the analysis with a presentation of the structure after equilibration as shown in Figure 2. The structure maintains the alpha-helical secondary structure in most of the system. However, we observe that the stutter region unfolds locally even without any applied force. This indicates a reduced stability of the alpha-helical secondary structure in this region and clearly reveals the formation of a structural defect at the site of the stutter. This result suggests that the stutter may not only be a region in which alpha helices run in parallel as proposed earlier, but that the stutter region may actually represent a complete, albeit local, loss of the alpha-helical structure.

In order to gain better insight into the unfolding observed during equilibration, free-energy calculations are performed that allow us to sample a much longer time-scale than accessible to standard molecular dynamics. Figure 3 shows the free-energy profile of the stutter region compared with a reference case that represents a neighbouring alphahelix region in which no instability is expected. This allows us to investigate the free-energy levels for an 'unfolded' and 'folded' state. In the 'folded' state, the end-to-end distance for one turn of alpha helix (corresponding to four residues) is around $0.6 \mathrm{~nm}$. In the 'unfolded' state, this distance fluctuates around $1.2 \mathrm{~nm}$, as shown in Figure 3(C).

For each case (stutter and reference), two different test systems are run, starting from the initial state of 'perfect' alpha helix and the protein where the stutter region has readily unfolded during equilibration. Figure 3(A) shows the free-energy profile for the stutter region. In both cases, the well-tempered metadynamics simulation visits two distinct states, 'folded' and 'unfolded'. The result confirms that the free energy associated with the 'unfolded' state (as also shown in the inset where the specific residue is marked) is lower than for the 'folded state'. The same analysis was performed for the reference case (Figure 

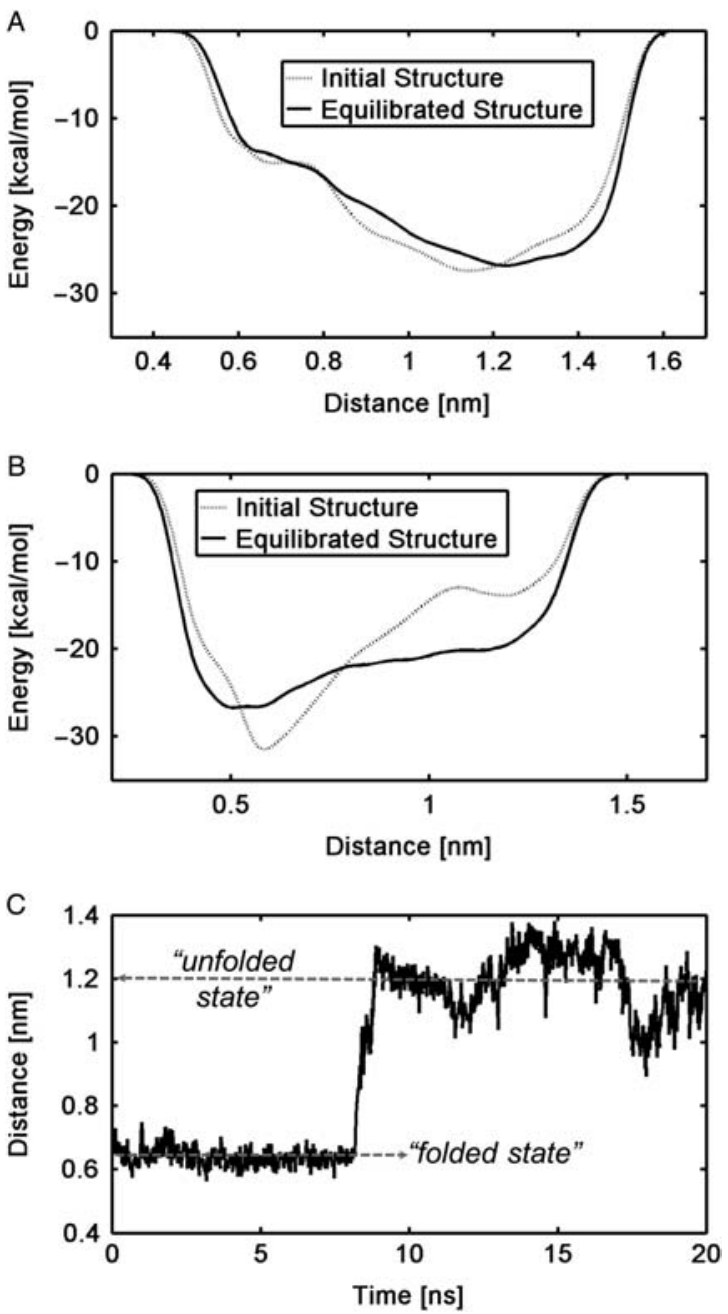

Figure 3. Metadynamics analysis of the free-energy landscape of the stutter (A) and a reference region with perfect alpha-helical coiled coils (B). Panel (A) shows the free-energy profile for the stutter region. The $x$-axis of the plot denotes the distance of one turn (residues 349-353) where the stutter exists. The free-energy landscape shows a minimum energy configuration at the 'unfolded' state, suggesting that it is more stable. Panel (B) shows the free-energy profile for the distance of one turn that constitutes residues 363-367 in the reference region. The results reveal that the minimum of the free-energy profile corresponds to the folded state for the alpha helix. Panel (C) shows the $20 \mathrm{~ns}$ equilibration result revealing the distances associated with the 'folded' (around $0.6 \mathrm{~nm}$ distance) and unfolded (around $1.2 \mathrm{~nm}$

3(B)), and the results show a minimum energy level at the 'folded' state, in contrast to the stutter case.

In addition to the different minimum free-energy locations for each case, this analysis also portrays the difference in the energy barrier to go from a 'folded' state to an 'unfolded' state between two different sets of residues. The energy level difference to go from a 'folded' state to an 'unfolded' state being $\sim 7 \mathrm{kcal} / \mathrm{mol}$ for the stutter region (Figure 3(A)), and that for the reference case distance) states, respectively. is shown to be $\sim 11 \mathrm{kcal} / \mathrm{mol}$. (Figure $3(\mathrm{~B})$ ); for the analysis performed starting from an equilibrated state. We note that when the analysis is carried out for the initially 'unfolded' state, we observe that the 'folded' state that the stutter visits during the metadynamics run is a slightly different folded configuration of a perfect alpha helix. The well-tempered metadynamics analysis presented here provides a powerful tool in determining the free-energy landscape associated with different states of the lengths of the regions of vimentin coil2, and confirms that the stutter region readily unfolds during equilibration because its free energy in the unfolded state is lower.

We now proceed with a presentation of a detailed nanomechanical analysis of the coil2 domain. Figure 4 shows force-displacement curve for the structure. In agreement with earlier studies (Ackbarow and Buehler 2009; Bertaud et al. 2010), we find that the curve has two distinct regions, where the force increases linearly until the structure is aligned with the pulling axis and followed by a small regime of axial stretching. The alignment of the structure with the pulling axis occurs at approximately $2 \mathrm{~nm}$ displacement, which is equivalent to about $10 \%$ strain for the $21 \mathrm{~nm}$ long structure (it is noted that the structure is not aligned with any orthogonal axis system after equilibration as seen in Figure 2).

After the initial almost linear-elastic region of the force-displacement curve in Figure 4 the angular point is reached, where a further increase in the prescribed displacement does not result in an increase in the force. This is due to the subsequent unfolding events that involve rupture of $\mathrm{H}$-bonds and as a result, alpha-helical turns that define the structure of the alpha helices. We observe that rupture of alpha-helical turns begins at the stutter and in the vicinity of the stutter region, as well as at the linker (L2) part at a displacement of about $4 \mathrm{~nm}$ (this implies a strain level of about $20 \%$ for the $21 \mathrm{~nm}$ long structure). This observation suggests that the initial defect formed at the stutter serves as a seed for the initiation of molecular unfolding under applied force. The data shown in Figure 4(A) suggest an average unfolding force of about $350 \mathrm{pN}$. For the pulling rate of $1 \mathrm{~m} / \mathrm{s}$ used here, this unfolding force is in accordance with the results from the previous studies (Bertaud et al. 2010). Figure 4(B) depicts the results of the same analysis performed over a range of different pulling rates. It is shown that the trend of initial linear-elastic region leads to a plateau over which the unfolding of different sections of the protein happens over a range of different pulling rates $(0.5,1,5$ and $10 \mathrm{~m} / \mathrm{s})$. However, the plateau force increases with increasing pulling rate as studied and shown in earlier work for protein unfolding simulations in explicit water (Bertaud et al. 2010).

Figure 5 displays a detailed analysis of chemical and structural changes during deformation, based on the percentage of change of the $\mathrm{H}$-bonds in the backbone of the structure as well as the secondary structure transition 
C

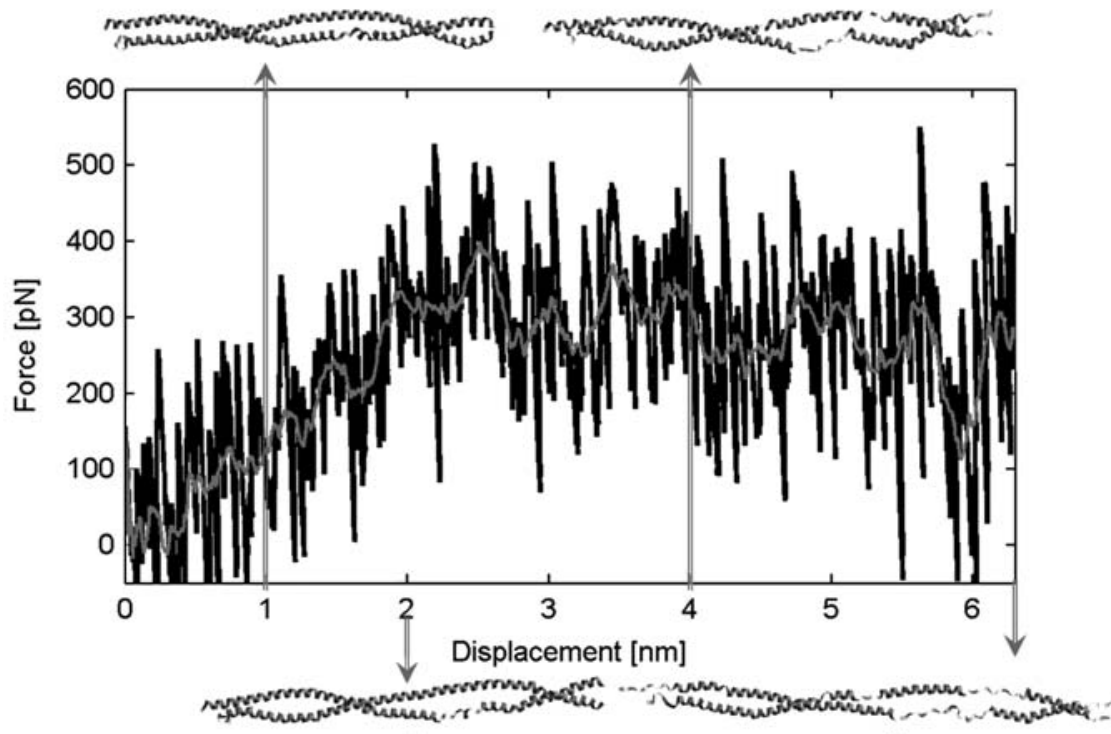

II

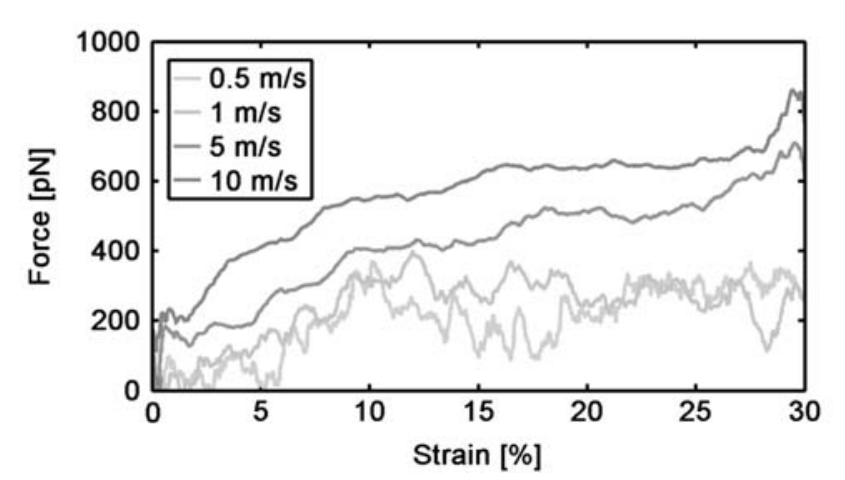

Figure 4. (I) Force-displacement curve of the coil2 structure, showing (A) at a displacement of $1 \mathrm{~nm}$, the structure is still aligned with the pulling axis and the stutter region is readily unfolded from the initial alpha helix form, (B) further increase in displacement results in alignment with the pulling axis as well as further unfolding of the stutter region which also defines the unfolding force (about $350 \mathrm{pN}$ ) which is constant throughout the subsequent unfolding events, (C) at a displacement of about $4 \mathrm{~nm}$, the linker region starts unfolding as well as the $2 \mathrm{~B}$ region, (D) further increase in displacement results in an unfolded $2 \mathrm{~B}$ region and an unstable linker region whereas the $2 \mathrm{~A}$ regions retain its form (i.e. alpha-helix configuration). (II) Force-displacement plots of tensile test simulations for four different pulling velocities $(0.5,1,5$ and $10 \mathrm{~m} / \mathrm{s})$. The plateau region depicting the consecutive unfolding events is at a significantly higher force level for higher pulling speeds such as 5 or $10 \mathrm{~m} / \mathrm{s}$.

due to the unfolding of the alpha-helix domains. Figure 5(A) shows the plot where the H-bonds breakage events are monitored in the duration of equilibration of the structure (20 ns). The negative change in the percentage of the H-bonds indicates the unfolding of the alpha helix along the backbone of the structure. A much higher ratio (about $25 \%$ ) of $\mathrm{H}$-bond rupture is observed as expected when the deformation is applied (Figure 5(B)). Here, again, the trend of the protein unfolding is dictated by the trend in the $2 \mathrm{~B}$ region of the protein. This assures us that there are no significant unfolding events that occur in the $2 \mathrm{~A}$ section of the structure up to strains of $30 \%$. Figure the unfolding of the alpha-helix domains with the applied strain, in agreement with the data provided in Figure 4.

\section{Conclusion}

We presented an explicit water molecular simulation analysis of the deformation of a $21 \mathrm{~nm}$ long vimentin coil2 domain and observed several striking results. Most importantly, we found that the stutter region readily unfolds even under the absence of any externally applied force, suggesting that the stutter is not very stable in the alpha-helical state. We confirmed this hypothesis using well-tempered metadynamics, which enabled us to obtain the free-energy configuration for an 'unfolded' and a 

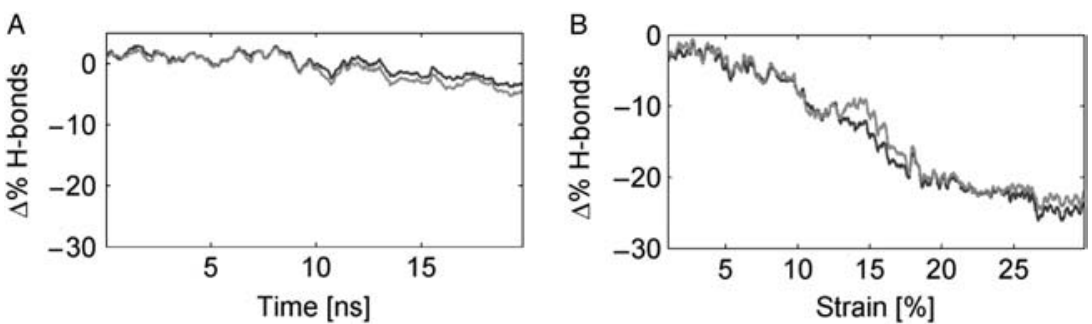

Coil (including segments 2A, L2 and 2B)

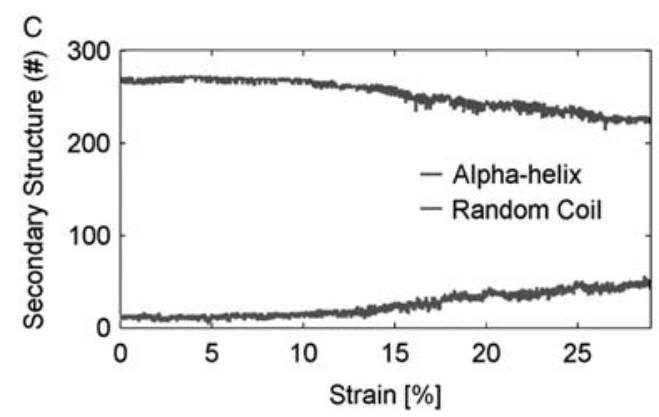

Figure 5. Percentage change in the number of backbone H-bonds in the duration of (A) equilibration (20 ns) and (B) deformation (plotted over the macroscopic strain of 30\%). In panel (A), the breaking of the H-bonds is observed during equilibration (amounting to about $5 \%$ ), the trend in the decrease of the percentage change in the number of H-bonds for the structure (the blue curve) is the same as that of segment $2 \mathrm{~B}$ (red curve). This implies that the breaking of the $\mathrm{H}$-bonds happens in segment $2 \mathrm{~B}$. Panel (B) shows that there is a nonlinear decrease (to up to about $25 \%$ change in the amount of the H-bonds) in the breakage of the H-bonds during deformation (the breakage is slower as the deformation reaches about $25 \%$ strain). Here, we can observe that the trend of H-bonds breakage is governed by segment 2B which experiences consecutive unfolding events. Note that in panel (B), the reference configuration is the one at the end of equilibrium. Panel (C) shows the secondary structure content as a function of strain for the entire coil2 (2A, L2 and 2B). As the strain increases, the number of alpha-helix domains decreases whereas the number of random coil domains (which do not correspond to a specific secondary structure) increases. This point at approximately $15 \%$ strain marks the occurrence of the unfolding events and the complete breakdown of the initial alpha-helical geometry.

'folded' state for the stutter region compared with a regular alpha-helical region that serves as a reference case. The analysis of these results highlighted that the stutter region is more stable in the 'unfolded' state, in agreement with the observations made in regular molecular dynamics simulations (Figure 3). By comparison, the analysis of the reference case confirms that the 'folded' state is more stable.

The thinner cross section of the stutter region represents a weak point as it forms a location with a reduced local bending stiffness. This could affect the persistence length along the strands which then affects the mobility of the structure during equilibration. Possible biological implications of this defined local unfolding may relate to signalling processes, where large changes in the molecular conformation may induce biochemical signalling cascades to indicate mechanical strain in varied cell mechanical states. A detailed analysis of these issues and further experimental validation of the predictions made here are left for future work.

Another interesting outcome of the simulation results presented here is the relative rigidity of the $2 \mathrm{~A}$ region, as anticipated earlier (Qin et al. 2009). The 2A region retains its alpha-helix form even up to a strain of $30 \%$ (at a displacement of about $6 \mathrm{~nm}$ ), which shows the relatively stiff nature of this region in the dimer. The work presented here is a part of a larger effort to elucidate structureproperty relationships in hierarchical materials, an effort defined as materiomics that could eventually result in an improved understanding of material factors in physiological conditions and disease (Cranford and Buehler 2010).

\section{Acknowledgements}

This work was supported by DOD-MURI, AFOSR and a PECASE grant. Additional support from NSF is acknowledged.

\section{References}

Ackbarow T, Buehler MJ. 2009. Molecular mechanics of stutter defects in vimentin intermediate filaments. Exp Mech. 49(1):79-89.

Ackbarow T, Sen D, Thaulow C, Buehler MJ. 2009. Alphahelical protein networks are self protective and flaw tolerant. PLoS ONE. 4(6):e6015.

Alberts B. 2002. Molecular biology of the cell. New York: Taylor and Francis. 
Barducci A, Bussi G, Parrinello M. 2008. Well-tempered metadynamics: a smoothly converging and tunable freeenergy method. Phys Rev Lett. 100(2):020603.

Bertaud J, Hester J, Jimenez DD, Buehler MJ. 2010. Energy landscape, structure and rate effects on strength properties of alpha-helical proteins. J Phys: Condens Matter. 22:035102.

Bonomi M, Branduardi D, Bussi G, Camilloni C, Provasi D, Raiteri P, Donadio D, Marinelli F, Pietrucci F, Broglia RA, Parrinello M. 2009. PLUMED: a portable plugin for freeenergy calculations with molecular dynamics. Comput Phys Commun. 180(10):1961-1972.

Q4 Brenner M, Johnson AB, Boespflug-Tanguy O, Rodriguez D, Goldman JE, Messing A. 2001. Mutations in GFAP, encoding glial fibrillary acidic protein, are associated with Alexander disease. Nat Genet. 27(1):117-120.

Brown JH, Cohen C, Parry DAD. 1996. Heptad breaks in alphahelical coiled coils: stutters and stammers. Proteins-Struct Funct Genet. 26(2):134-145.

Buehler MJ, Ackbarow T. 2008. Nanomechanical strength mechanisms of hierarchical biological materials and tissues. Comput Methods Biomech Biomed Engin. 11(6):595-607.

Buehler MJ, Yung YC. 2009. Deformation and failure of protein materials in physiologically extreme conditions and disease. Nat Mater. 8(3):175-188.

Cranford SW, Buehler MJ. 2010. Materiomics: biological protein materials, from nano to macro. Nanotechnol Sci Appl. 3:127-148.

Dahl KN, Kahn SM, Wilson KL, Discher DE. 2004. The nuclear envelope lamina network has elasticity and a compressibility limit suggestive of a molecular shock absorber. J Cell Sci. 117(20):4779-4786.

Fudge D, Russell D, Beriault D, Moore W, Lane EB, Vogl AW. 2008. The intermediate filament network in cultured human keratinocytes is remarkably extensible and resilient. PLoS ONE. 3(6):e2327.

Herrmann H, Aebi U. 2004. Intermediate filaments: molecular structure, assembly mechanism, and integration into functionally distinct intracellular scaffolds. Annu Rev Biochem. 73:749-789.

Herrmann H, Bar H, Kreplak L, Strelkov SV, Aebi U. 2007. Intermediate filaments: from cell architecture to nanomechanics. Nat Rev Mol Cell Biol. 8(7):562-573.

Humphrey W, Dalke A, Schulten K. 1996. VMD: visual molecular dynamics. J Mol Graph. 14(1):33-38.

Hutchison CJ. 2002. Lamins: building blocks or regulators of gene expression? Nat Rev Mol Cell Biol. 3(11):848-858.

Kreplak L, Herrmann H, Aebi U. 2008. Tensile properties of single desmin intermediate filaments. Biophys J. 94(7):2790-2799.

Lewis MK, Nahirney PC, Chen V, Adhikari BB, Wright J, Reedy MK, Bass AH, Wang K. 2003. Concentric intermediate filament lattice links to specialized Z-band junctional complexes in sonic muscle fibers of the type I male midshipman fish. J Struct Biol. 143(1):56-71.

Lu H, Isralewitz B, Krammer A, Vogel V, Schulten K. 1998. Unfolding of titin immunoglobulin domains by steered molecular dynamics simulation. Biophys J. 75(2):662-671.

MacCallum JL, Moghaddam MS, Chan HS, Tieleman DP. 2007. Hydrophobic association of alpha-helices, steric dewetting, and enthalpic barriers to protein folding. Proc Natl Acad Sci USA. 104(15):6206-6210.

MacKerell AD et al. 1998. All-atom empirical potential for molecular modeling and dynamics studies of proteins. J Phys

Q7 Chem B. 102(18):3586-3616.

Nelson MT, Humphrey W, Gursoy A, Dalke A, Kalé LV, Skeel RD, Schulten K. 1996. NAMD: a parallel, object oriented moleculare dynamics program. Int J Supercomput Appl High Perform Comput. 10(4):251-268.

Nicolet S, Herrmann H, Aebi U, Strelkov SV. 2010. Atomic Q6 structure of vimentin coil 2. J Struct Biol. 170(2):369-376.

Omary MB, Coulombe PA, McLean WH. 2004. Intermediate filament proteins and their associated diseases. N Engl J Med. 351(20):2087-2100.

Parry DAD, Strelkov SV, Burkhard P, Aebi U, Herrmann H. 2007. Towards a molecular description of intermediate filament structure and assembly. Exp Cell Res. 313(10):2204-2216.

Q5 Qin Z, Kreplak L, Buehler MJ. 2009. Nanomechanical properties of vimentin intermediate filament dimers. Nanotechnology. 20(42):425101.

Rose A, Meier I. 2004. Scaffolds, levers, rods and springs: diverse cellular functions of long coiled-coil proteins. Cell Mol Life Sci. 61(16):1996-2009.

Strellkov S. 2002. Conserved segments $1 \mathrm{~A}$ and $2 \mathrm{~B}$ of the intermediate filament dimer: their atomic structures and role in filament assembly. EMBO J. 21(6):1255-1266.

Strelkov SV, Herrmann H, Geisler N, Lustig A, Ivaninskii S, Zimbelmann R, Burkhard P, Aebi U. 2001. Divide-andconquer crystallographic approach towards an atomic structure of intermediate filaments. $\mathrm{J}$ Mol Biol. 306(4):773-781.

Strelkov SV, Herrmann H, Geisler N, Wedig T, Zimbelmann R, Aebi U, Burkhard P. 2002. Conserved segments 1A and 2B of the intermediate filament dimer: their atomic structures and role in filament assembly. EMBO J. 21(6):1255-1266.

Strelkov SV, Herrmann H, Aebi U. 2003. Molecular architecture of intermediate filaments. Bioessays. 25(3):243-251.

Wang N, Butler JP, Ingber DE. 1993. Mechanotransduction across the cell surface and through the cytoskeleton. Science. 260(5111):1124-1127.

Wang N, Stamenovic D. 2002. Mechanics of vimentin intermediate filaments. J Muscle Res Cell Motil. 23(56):535-540.

Wilson KL, Zastrow MS, Lee KK. 2001. Lamins and disease: insights into nuclear infrastructure. Cell. 104(5):647-650. 